\title{
Short report \\ Cellulitis in liver cirrhosis - a series of 25 cases from southern India
}

\author{
Uday Sanglodkar, Mayank Jain, Dinesh Jothimoni, Subhashree Parida, Balajee G, Jayanthi Venkataraman
}

Liver and Gastroenterology Units, Gleneagles Global Health City, Chennai, India

\begin{abstract}
Introduction: Cirrhosis of the liver predisposes patients to serious bacterial infections including cellulitis. The aim of the study was to determine the clinical and bacteriological profile of cellulitis in patients with liver cirrhosis.

Material and methods: In this prospective study, consecutive cirrhotic patients hospitalized between February and September 2017 were enrolled. Data retrieved included demographics, aetiology, co-morbidity, cirrhosisrelated complications, site of cellulitis, baseline investigations, and wound and blood culture.

Results: Three hundred and thirty-eight patients were admitted, of whom 25 had cellulitis (mean age $52.8 \pm$ 10.4 years, men $88.0 \%$, median MELD [model of end-stage liver disease] $18.8 \pm 10.4$ ). Non-alcoholic steatohepatitis and alcohol were each the cause of cirrhosis in $40 \%$ of cases. Nine patients (36\%) had diabetes mellitus. Cultures were positive in 13 cases (52\%; 1 - blood, 11 - wound, 1 - both blood and skin). The majority of the cultures showed monomicrobial growth $(9 ; 70 \%)$. Escherichia coli was the commonest isolate $(6 / 13)$, followed by Klebsiella (4). $61 \%$ of isolates had multidrug resistant organisms. The outcome was favourable.

Conclusions: Prevalence of cellulitis was 7.4\% in hospitalised cirrhotics. Culture positivity was 52\%. Escherichia coli and Klebsiella were the most common isolates and three fifths of isolates were multidrug resistant.
\end{abstract}

Key words: infection, alcoholism, chronic liver disease.

\section{Address for correspondence}

Dr. Mayank Jain, Gleneagles Global Health City, 439 Cheran Nagar, 600100 Chennai, India, phone: 917312365688 e-mail: mayank4670@rediffmail.com

\section{Introduction}

Cirrhosis of the liver predisposes patients to serious bacterial infections including cellulitis [1]. These infections precipitate decompensation of liver disease and lead to increased morbidity and mortality [2]. Multiple immune system defects such as complement deficiency, reduced chemo-attractant activity, decreased polymorphonuclear leukocyte activity, and reduced number of Kupffer cells predispose cirrhotics to these infections [3].

In an earlier study from our centre, we reported multidrug resistance in $69 \%$ of bacterial isolates in cirrhotics [4]. The present study was undertaken to determine the clinical and bacteriological profile of skin infection presenting as cellulitis in patients with liver cirrhosis.

\section{Material and methods}

The present study was conducted at the Liver and Gastroenterology Units of Gleneagles Global Health City, Chennai. In this prospective study, consecutive cirrhotic patients hospitalized between February 2017 and September 2017 with the clinical diagnosis of cellulitis were enrolled. Data gathered included demographics, aetiology of cirrhosis, co-morbidity, cirrhosis-related complications, site of cellulitis, baseline investigations, and wound and blood culture reports with antibiotic sensitivity. As per the institution protocol, all patients were empirically started on piperacillin-tazobactam until results of cultures were available and then the antibiotics were subsequently modified. Details of duration of antibiotic treatment and outcome were recorded. This was a single point 
study and the outcome at the time of discharge was noted.

Informed consent was taken from all the patients and the protocol was approved by the Institutional Ethics Committee.

\section{Results}

\section{Baseline characteristics}

Three hundred and thirty-eight patients with end stage liver disease were admitted during the study period. Of these, 25 patients (7.4\%) had cellulitis and they constituted the study cohort. The median age was $52.8 \pm 10.4$ years. The majority were men (88.0\%). The median MELD [model of end-stage liver disease] score was 18.8 (range 11-32). Non-alcoholic steatohepatitis (10) and alcohol (10) contributed to $40 \%$ of cirrhosis followed by cryptogenic disease $(4,16 \%)$ and hepatitis B $(1,4 \%)$. Nine patients (36\%) had type 2 diabetes mellitus. There was only 1 past smoker. Apart from cellulitis, other complications during admission included refractory ascites and renal dysfunction in $36 \%$ (9 cases) respectively, spontaneous bacterial peritonitis in $12 \%$ (3 cases), hepatic encephalopathy and gastrointestinal bleeding in $8 \%$ (2 cases) respectively. Three (12\%) of the 25 patients had cellulitis in the past. The baseline haematological, biochemical and endoscopic parameters at recruitment are shown in Table 1.

\section{Skin involvement (Figure 1)}

The majority of the patients $(17,68 \%)$ had unilateral or bilateral cellulitis presenting as painful lower limb

Table 1. Baseline haematological, biochemical and endoscopic parameters of the study cohort

\begin{tabular}{|c|c|c|}
\hline Parameters & Median & Range \\
\hline Body mass index $\left(\mathrm{kg} / \mathrm{m}^{2}\right)$ & 27.9 & $20.8-32.5$ \\
\hline Haemoglobin (gm/dl) & 8.83 & $8.1-10.1$ \\
\hline Total leucocyte count (cells $/ \mathrm{m}^{2}$ ) & 18779 & $5980-35801$ \\
\hline Platelets (cells/m³) & 102000 & $19451-161000$ \\
\hline Serum bilirubin (mg/dl) & 6.05 & $2.18-35.64$ \\
\hline Serum creatinine (mg/dl) & 1.5 & $0.5-3.1$ \\
\hline INR (international normalized ratio) & 1.67 & $1.09-3.48$ \\
\hline Serum albumin (gm/dl) & 2.45 & $1.7-3.1$ \\
\hline Oesophageal varices & $\begin{array}{c}\text { Grade } 3-6 \text { cases } \\
\text { Grade } 2-18 \text { cases } \\
\text { Grade } 1-1 \text { case }\end{array}$ & \\
\hline $\begin{array}{l}\text { Prior history of endoscopic } \\
\text { variceal ligation }\end{array}$ & 20 cases & \\
\hline
\end{tabular}

oedema $(5,20 \%)$. Two patients had unilateral upper limb involvement $(2,8 \%)$ and scrotal oedema was noted in 1 case (4\%). The skin was erythematous and inflamed with warmth and tenderness on touch. Fifteen patients $(60 \%)$ had superficial ulcerated lesions and serous fluid ooze from the superficial lesion; one patient had bullous lesion. In eight cases, Doppler study of the lower limbs was done to rule out suspected arterial and venous thrombosis. However, in none of the cases, this could be established.

\section{Cultures and antibiotic sensitivity}

Culture positivity was noted in thirteen cases (52\%; 1 - blood, 11 - discharge from the affected skin, 1 - both blood and skin). The majority of the cultures showed monomicrobial growth $(9,70 \%)$ and the remainder were polymicrobial. Escherichia coli was the commonest isolate (6/13), followed by Klebsiella (4), Pseudomonas (2) and Staphylococcus aureus (2). Eight isolates (61\%) had multidrug resistant organisms (ESBL [extended-spectrum beta-lactamases] - 6, carbapenemase-producing Klebsiella - 2). Average duration of treatment was 10 days ( \pm 4.1 days). Twenty-two patients were discharged with healing of the wound; 2 left against medical advice and 1 died due to multiorgan failure.

\section{Discussion}

Cellulitis in cirrhosis is being increasingly reported. A nationwide population-based study in Taiwan showed that cirrhotic patients are at higher risk of cellulitis than non-cirrhotics [5]. Studies from India and across the world have reported the prevalence to vary between 6.7 and 21\% (Table 2). In the present series, we found that $7.4 \%$ of our admitted patients had cellulitis.

Cellulitis in liver cirrhosis is caused by numerous factors. Increased bacterial translocation from the gut due to altered intestinal immunity and bacterial overgrowth in cirrhotics produces Gram-negative bacteraemia, giving an opportunity for distant invasion by these microorganisms [3]. This is substantiated by the culture reports in these patients [6-8]. Moreover, low albumin levels are also a risk factor for skin infection $[6,11]$. With advanced liver disease, poor liver function results in increased susceptibility to infection due to defective bactericidal function of immunoglobulin, decreased polymorphonuclear leukocyte activity, complement deficiency, and a reduced number of Kupffer cells [12]. Chronic oedema, congestion and barefoot walking are other risk factors for cellulitis in cirrhotics $[6,10]$. 

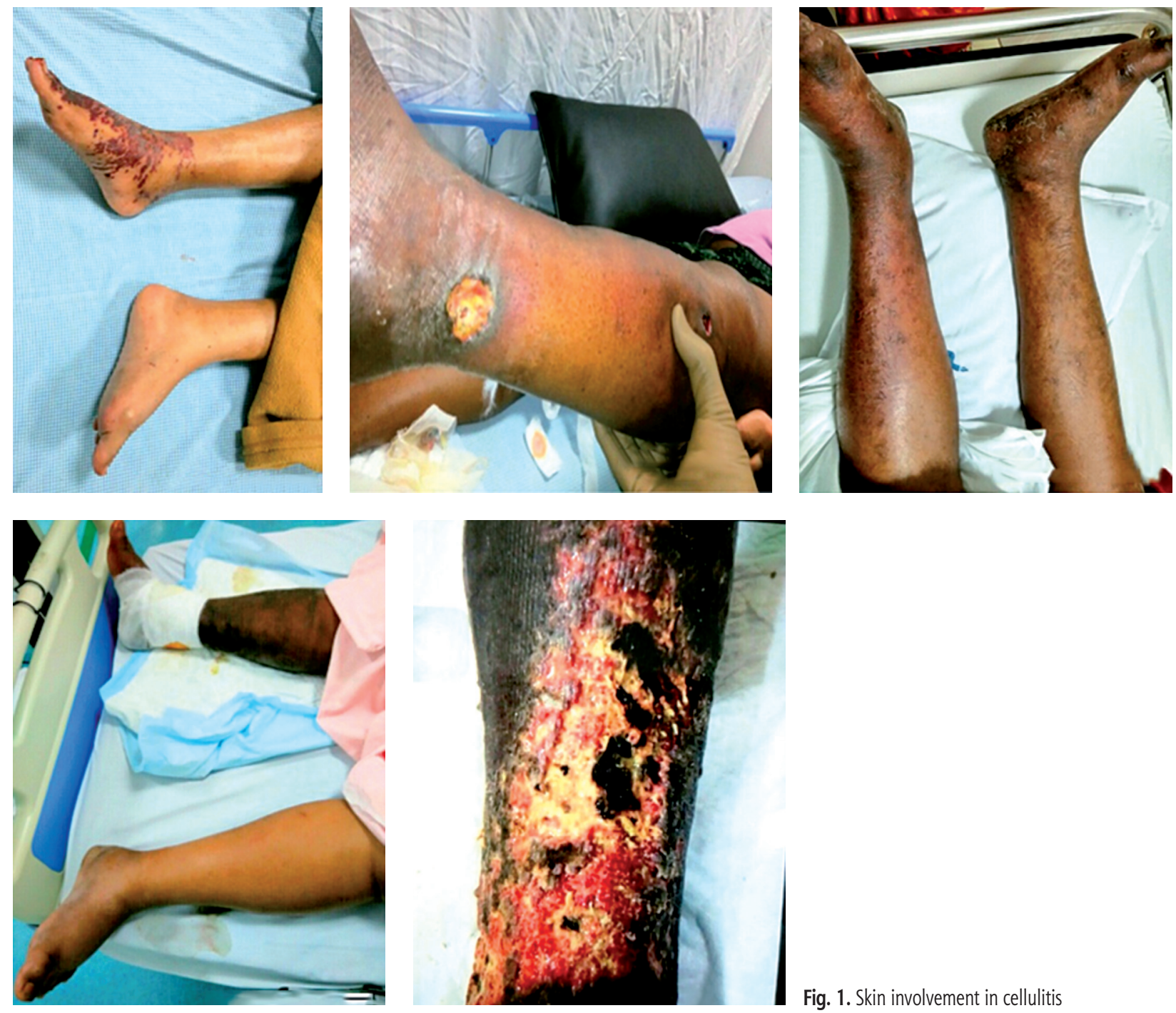

Fig. 1. Skin involvement in cellulitis

We noted that the majority of patients had lower limb involvement and only a few had isolated upper limb or scrotal involvement. Three fifth of patients had superficial ulcers.
In cirrhotic patients, Gram-negative bacteria (GNB), mainly Escherichia coli, predominate in SBP and urinary tract infections, while Gram-positive cocci (GPC) predominate in patients with pneumonia and

Table 2. Studies on skin infection in end stage liver disease

\begin{tabular}{|c|c|c|c|c|c|}
\hline Study & No. of patients & Aetiology for cirrhosis & Prevalence of skin infections & Culture isolates & Mortality \\
\hline Mohan et al. [6] & 200 & Alcohol & $21 \%$ & $\begin{array}{l}\text { GNB } 15 / 17 \\
\text { GPC } 2 / 17\end{array}$ & $19 \%$ \\
\hline Jain et al. [7] & 103 & Alcohol & $8.7 \%$ & $\begin{array}{l}\text { GNB 5/9 } \\
\text { GPC 4/9 }\end{array}$ & $22.2 \%$ \\
\hline Sood et al. [8] & 1395 & Alcohol & $6.37 \%$ & $\begin{array}{l}\text { GNB } 13 / 15 \\
\text { GPC } 2 / 15\end{array}$ & $23.5 \%$ \\
\hline Kim et al. [9] & 80 & - & $12.5 \%$ & - & - \\
\hline Lin et al. [5] & 39966 & Alcohol, diabetes & $6.7 \%$ & - & - \\
\hline Present study & 338 & $\begin{array}{l}\text { Alcohol, NASH, } \\
\text { cryptogenic }\end{array}$ & $7.4 \%$ & $\begin{array}{l}\text { GNB } 14 / 17 \\
\text { GPC } 3 / 17\end{array}$ & $4 \%$ \\
\hline
\end{tabular}

NASH - non-alcoholic steatohepatitis, GNB - Gram-negative bacteria, GPC - Gram-positive cocci 


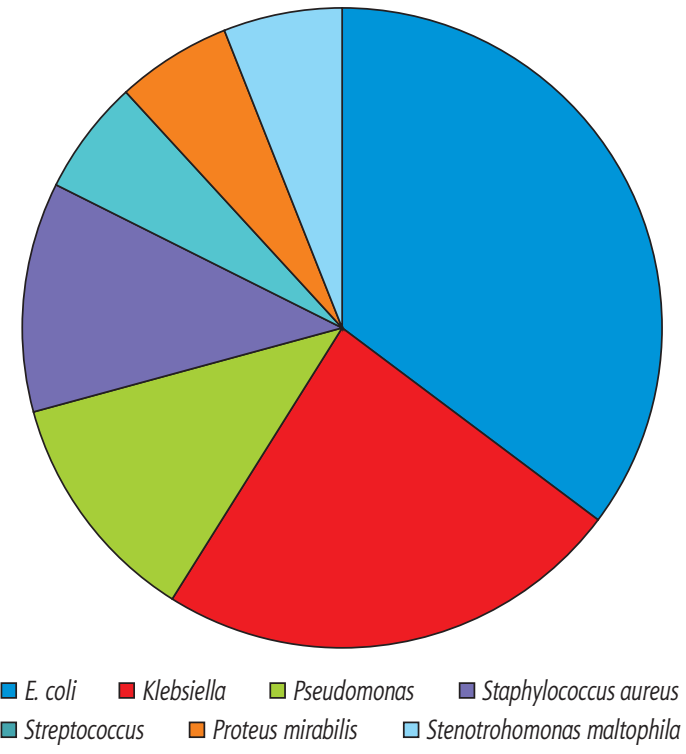

Fig. 2. Bacterial isolates in patients with cellulitis

bacteraemia associated with invasive procedures [13]. Our observations were similar to the Gram-negative isolates from skin in other studies [6-8] (Table 2).

The mortality rate in cirrhotics with skin infections varies from $19 \%$ to $23.5 \%$, depending on disease severity [6-8]. Haemorrhagic bullae and Child-Pugh grade C have been reported as possible predictors of mortality in cirrhotics with skin infection [13-15]. Serum creatinine and MELD score have been found to be independent predictors of mortality in these patients [7]. In our cohort, only 1 patient with a MELD score of 32, renal dysfunction and bacteraemia died during the hospital stay. The majority of the remaining cases responded well to appropriate antibiotics.

Since most of the infections are caused by Gramnegative organisms, the treatment of choice is broad spectrum antibiotic therapy. We noted that $61.5 \%$ of the isolates had multidrug resistant organisms and required imipenem, colistin or tigecycline for treatment. This is an alarming finding. Our centre is a referral centre for liver transplantation and the majority of our cases are referred at an advanced stage for further management. The majority of them receive numerous antibiotics for infective complications at other centres, and this may be responsible for the high incidence of multidrug resistant isolates at our centre.

Our study was a single point study and we did not follow up cases. However, recurrence of infection has been reported in about a fifth of patients within six months [11] of the first infection. Three of our patients gave a history of previous skin infection/cellulitis. In a study from South India, use of prophylactic antibiotics was found to reduce the rate of cellulitis recurrence [11]. However, in the absence of large studies, this prophylaxis is not universally recommended. Nevertheless, this is likely to be worthwhile in patients with advanced liver disease, MELD > 15 and those with recurrent cellulitis.

\section{Conclusions}

Prevalence of cellulitis in hospitalised cirrhotic patients at our centre was $7.4 \%$. Almost $50 \%$ of infections were due to a monomicrobial infection (Escherichia coli and Klebsiella) and three fifths of the isolates were multidrug resistant. Appropriate institution of antibiotics resulted in a favourable outcome, with one death.

\section{Disclosure}

The authors report no conflict of interest.

\section{References}

1. Gustot T, Durand F, Lebrec D, et al. Severe sepsis in cirrhosis. Hepatology 2009; 50: 2022-2033.

2. Cheruvattath R, Balan V. Infections in patients with end-stage liver disease. J Clin Gastroenterol 2007; 41: 403-411.

3. Tandon P, Garcia-Tsao G. Bacterial infections, sepsis and multiorgan failure in cirrhosis. Semin Liver Dis 2008; 28: 26-42.

4. Jain M, Varghese J, Michael T, et al. An Insight into Antibiotic Resistance to Bacterial Infection in Chronic Liver Disease. J Clin Exper Hepatol 2017; 7: 305-309

5. Lin MN, Tsai CC, Hung TH, Tsai CC. The risk of cellulitis in cirrhotic patients: a nationwide population-based study in Taiwan. Gut Liver 2012; 6: 482-485.

6. Mohan P, Ramu B, Bhasker E, Venkataraman J. Prevalence and risk factors for bacterial skin infection and mortality in cirrhosis. Ann Hepatol 2011; 10: 15-20.

7. Jain $\mathrm{M}$, Waghmare $\mathrm{C}$, Adkar S, et al. Bacterial skin infections in cirrhotics. Indian J Gastroenterol 2014; 33: 92.

8. Sood A, Midha V, Goyal O, et al. Skin and soft tissue infections in cirrhotics: A prospective analysis of clinical presentation and factors affecting outcome. Indian J Gastroenterol 2014; 33: 281-284.

9. Kim JH, Lee JS, Lee SH, et al. Renal dysfunction induced by bacterial infection other than spontaneous bacterial peritonitis in patients with cirrhosis: incidence and risk factor. Gut Liver 2009; 3: 292-297.

10. Dupuy A, Benchikhi H, Roujeau JC, et al. Risk factors for erysipelas of the leg (cellulitis): case-control study. Br Med J 1999; 318: 1591-1594.

11. Hamza RE, Villyoth MP, Peter G, et al. Risk factors of cellulitis in cirrhosis and antibiotic prophylaxis in preventing recurrence. Ann Gastroenterol 2014; 27: 374-379.

12. Wyke RJ. Problems of bacterial infection in patients with liver disease. Gut 1987; 28: 623-641.

13. Borzio M, Salerno F, Piantoni L, et al. Bacterial infection in patients with advanced cirrhosis: a multicentre prospective study. Dig Liver Dis 2001; 33: 41-48.

14. Liu BM, Chang KJ, Chen CH, et al. Risk factors for the outcome of cirrhotics patients with soft tissue infections. J Clin Gastroenterol 2008; 3: 312-316.

15. Liu BM, Hsiao CT, Chung KJ, et al. Haemorrhagic bullae represent an ominous sign for cirrhotic patients. J Emerg Med 2008; 34: 277-281. 